\title{
Comment on: Gale EAM (2007) To boldly go-or to go too boldly? The accelerator hypothesis revisited. Diabetologia 50:1571-1575-a reply to the editor
}

\author{
T. J. Wilkin
}

Received: 11 July 2007 / Accepted: 8 August 2007 / Published online: 5 October 2007

(C) Springer-Verlag 2007

Keywords Accelerator hypothesis · Aetiopathogenesis · Classification · Debate, type 1 diabetes

To the Editor: The Editor's erudite commentary [1] on the accelerator hypothesis and its impact on the classification of diabetes [2] points to where the hypothesis may be vulnerable, offers the opportunity for adjustment and sets an intellectual standard by which the hypothesis will stand or fall. The hypothesis is based on the construct of tempo [3], and envisages a hierarchy of genotypes that respond to insulin resistance with corresponding rates of beta cell loss. Accordingly, a rise in insulin resistance across a population would predict a leftward shift, resulting in younger age at onset and an increase in incidence, both fundamental tenets of the hypothesis because they spell acceleration.

Constitution predisposes to accelerated apoptosis Gale's expectation from the accelerator hypothesis is that 'both forms of diabetes share a common genetic susceptibility to beta cell loss'. But this is to misconstrue the hypothesis ('Type 1 and type 2 diabetes are the same disorder of insulin resistance set against different genetic backgrounds'). The hypothesis does not propose a common genetic soil, but rather a common environmental risk-insulin resistance-to which different genotypes respond in a hierarchical fashion. I have responded to this point elsewhere [4], but an analogy may help to clarify it. If the fish in a lake were 'accelerator' genes encoding susceptibility according to their size, only the widest of meshes (the

T. J. Wilkin $(\bowtie)$

University Medicine, Level 7, Derriford Hospital,

Plymouth PL6 8DH, UK

e-mail: T.Wilkin@pms.ac.uk least insulin resistance) would be needed to net the largest of them, i.e. those (HLA) genes associated with fast tempo diabetes (type 1). Smaller fish would not be selected by the trawl, and would not find themselves partitioned one side of a net designed to catch type 1 fish. These smaller fish would be trapped only with a narrower mesh, i.e. the genes associated with type 2 diabetes would be expressed only when insulin resistance was greater. I would therefore expect to find type 1 genes overexpressed in type 2 diabetes, but not type 2 genes, such as TCF7L2, in type 1 diabetes, as Field et al. observed [5]. FTO is not an accelerator gene, but a determinant of body mass that is over-represented in type 2 diabetes, where BMI surfeits are large, but not in type 1 [6] because, although corresponding surfeits are consistently reported [2], they are smaller.

Insulin resistance is the principal cause of non-immune diabetes I recognise that insulin resistance and beta cell performance are inter-dependent functions in a non-linear loop, but the question at issue is whether the same insulin resistance that most would accept underpins type 2 diabetes also underpins type 1 . The two examples in the literature which I cited to suggest that it might, both adjusted their measure of insulin resistance for beta cell reserve, as Gale requires. Both reported that those who went on to type 1 diabetes were more insulin resistant relative to, in the first instance, their identical twin who did not [7] and, in the second, those in the same randomised cohort who did not [8]. I think both satisfy Gale's concern for 'relative to what?' Everybody is, by degrees, insulin deficient, and the hypothesis argues that the tempo of progression from health to diabetes moves along a continuum, not from one arbitrary category to another.

Insulin sensitivity only becomes an important influence upon progression to hyperglycaemia once critical levels of 
secretory failure are present Gale cites three data sets as evidence that the influence of insulin resistance on disease progression is limited to a sub-set whose beta cell failure is already far advanced. However, such categorisation of what is biologically a continuous process may be misleading. The median observation periods for the Diabetes Prevention Trial 1 (DPT-1; 3.7 years) [9], the European Nicotinamide Diabetes Intervention Trial (ENDIT; 4.2 years) [10] and the SEARCH for Diabetes in Youth study ( $<4$ years) [11] were brief, whereas the accelerator hypothesis attempts to explain the emergence of diabetes over a lifetime. Those individuals in the three studies whose C-peptide or first-phase insulin response was preserved were, by implication, of slower tempo than those in whom it was already lost. Because of their short duration, the studies may have lacked the numbers to detect an association between insulin resistance and future diabetes in the group with preserved FPIR in whom the tempo was slowest and the risk of diabetes lowest. Many decades may be needed to reveal the relationship between BMI and age at onset where the tempo is slow [12]. The four studies that have unequivocally reported an inverse relationship between age at onset of type 1 diabetes and BMI observed whole populations over periods of 20 years or more [2], rather than selected subjects over fewer than 5 years. It is brave to conclude on the basis of such shortterm studies that 'decreasing insulin sensitivity...has no causal role in the pathogenesis of type 1 diabetes' or to predict, without first testing the possibility, that insulin sensitisation could do no more than 'postpone the need for insulin by a few months'.

Does the hypothesis fit the facts? Putting the more philosophical arguments of insulin insensitivity vs insulin resistance to one side, height velocity, weight gain, puberty and pregnancy are all physiological processes associated with insulin resistance. All are accelerators of beta cell failure and all are associated with an increased incidence of type 1 diabetes. Perhaps the strongest evidence of a connection with insulin resistance is that BMI, alone among the many environmental factors proposed, shows an inverse correlation with age at onset-true acceleration. The observation suggests a dose-response relationship between insulin resistance and type 1 diabetes within individuals, not merely their co-existence among individuals.

The planned metformin intervention trial to test the accelerator hypothesis may reveal more than Gale concedes. If, as he predicts, there proves to be no useful effect, the accelerator hypothesis will fall, and with it that of overlap (double diabetes) as an alternative explanation for the rise in incidence of type 1 diabetes. If, as the investigators believe, the effect will be $30 \%$ or greater (a conservative estimate based on the use of metformin in the American Diabetes Prevention Program [DPP] [13]), the result will not be readily attributable to overlap, because type 2 diabetes does not account for $30 \%$ of childhood diabetes. If it were to be so attributed, the logic of doing so must conclude that type 1 and type 2 diabetes are one and the same disorder because $100 \%$ of study subjects will be primary relatives of people with classic type 1 diabetes who on entry to the trial will carry at least two of the three autoantibodies considered to be the hallmark of type 1 diabetes.

Finally, the Editor's commentary prompts some useful modification to the hypothesis. The premise remains unchanged, but if the tempo of beta cell loss reflects, essentially, the interplay between insulin resistance (beta cell stress) and responsive genes, then the accelerators can be reduced to these alone (see Fig. 1), where a genetic hierarchy fixes susceptibility of the beta cell to insulin resistance. Genes at one end of the continuum would afford the beta cell relative protection against the inflammation associated with

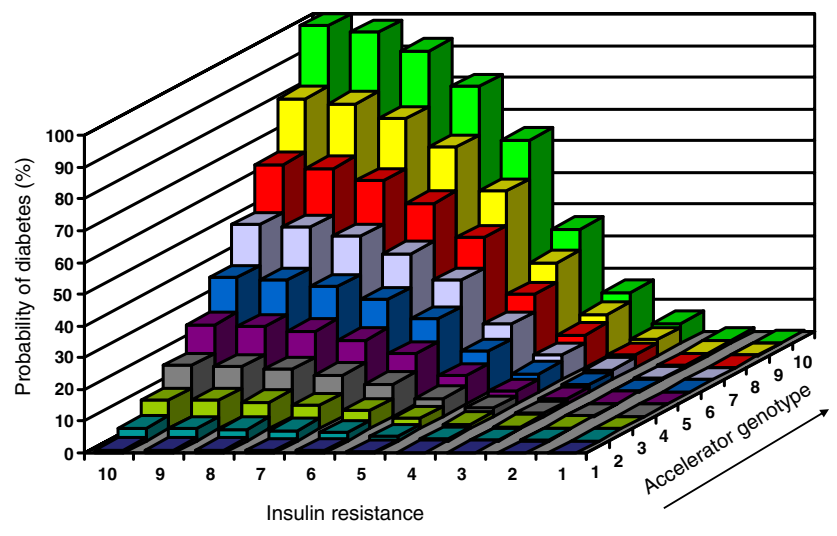

Fig. 1 Schematic representation of the (adjusted) accelerator hypothesis, where the interplay between two accelerators of beta cell loss - insulin resistance (risk) and accelerator genes (susceptibility) - determines the probability of multifactorial diabetes. A hierarchy of accelerator genes (or their combination) is envisaged, giving rise to the family of doseresponse curves (from front to back) in the figure. Diabetes is unlikely, whatever the level of insulin resistance, if there are few accelerator genes (consistent with the absence of diabetes in the majority of even the most obese). Diabetes is similarly unlikely, whatever the genotype (even the HLA genotype conferring highest susceptibility), if insulin resistance remains low (consistent with the absence of diabetes in the majority of those carrying high-risk genotypes). Genes that influence metabolic function of the beta cell (e.g. TCF7L2) are of a lower order, but part of the same accelerator gene hierarchy as HLA, which sit towards the top. The response to rising insulin resistance is sharpest among those who carry the most reactive accelerator genes, where only a modest increase is needed to raise the probability of diabetes substantially. Higher levels of insulin resistance, achieved nowadays by more children because of their obesity, bestow the same probability of diabetes with lower order accelerator genes (consistent with the documented fall in high-order susceptibility genes among contemporary children with type 1 diabetes). Autoimmunity is viewed by the hypothesis as the immune response to beta cells 'stressed' by insulin resistance. The increasing contribution made to the probability of diabetes by rising insulin resistance can explain why $\mathrm{T}$ cells reactive to islet-related antigens (classic features of type 1 diabetes) are now reported in children who are phenotypically type 2 and of a lower order genotype 
its metabolic upregulation. Genes at the other end - the HLA genes-would exacerbate the inflammation and greatly accelerate the tempo of beta cell loss.

As Gale points out, a hypothesis cannot be tested by observation. Intervention studies are needed, and a randomised controlled trial of metformin is planned, involving the Oxford Diabetes Trials Unit and an international panel of experts in type 1 diabetes under the auspices of the International Society for Pediatric and Adolescent Diabetes (ISPAD). An answer is a long way off, but an intervention of this kind should fulfil the mission of science generally, and that of Trekkies specifically: 'To boldly go...where no man has gone before.'

Acknowledgements I am grateful to B. Metcalf, senior statistician with the EarlyBird study, for his help and insights into constructing the figure.

Duality of interest The author declares that there is no duality of interest associated with this manuscript.

\section{References}

1. Gale EAM (2007) To boldly go - or to go too boldly? The accelerator hypothesis revisited. Diabetologia 50:1571-1575

2. Wilkin TJ (2007) Changing perspectives in diabetes: their impact on its classification. Diabetologia 50:1587-1592
3. Wilkin TJ (2001) The accelerator hypothesis: weight gain as the missing link between type I and type 11 diabetes. Diabetologia 44:914-922

4. Wilkin TJ (2007) The accelerator hypothesis cannot be tested using the type 2 diabetes gene, TCF7L2. Diabetologia 50:1780

5. Field SF, Howson JMM, Smyth DJ, Walker NM, Dunger DB, Todd JA (2007) Analysis of the type 2 diabetes gene, TCF7L2, in 13,795 type 1 diabetes cases and control subjects. Diabetologia 50:212-213

6. Field SF, Howson JMM, Walker NM, Dunger DB, Todd JA (2007) Analysis of the obesity gene FTO in 14,803 type 1 diabetes cases and controls. Diabetologia DOI 10.1007/s00125-007-0767-0

7. Hawa MI, Bonfanti R, Valeri C, Delli Castelli M, Beyan H, Leslie RD (2005) No evidence for genetically determined alteration in insulin secretion or sensitivity predisposing to type 1 diabetes: a study of identical twins. Diabetes Care 28:1415-1418

8. Fourlanos S, Narendran P, Byrnes GB, Colman PG, Harrison LC (2004) Insulin resistance is a risk factor for progression to type 1 diabetes. Diabetologia 47:1661-1667

9. Diabetes Prevention Trial-Type 1 Diabetes Study Group (2002) Effects of insulin on relatives of patients with type 1 diabetes. N Engl J Med 346:1658-1691

10. The European Nicotinamide Diabetes Intervention Trial (ENDIT) Group (2002) A randomised controlled trial of intervention before the onset of type 1 diabetes. Lancet 363:925-931

11. Dabelea D, D'Agostino RB, Mayer-Davis EJ et al (2006) Testing the accelerator hypothesis: body size, beta-cell function, and age at onset of type 1 (autoimmune) diabetes. Diabetes Care 29:290-294

12. Wilkin TJ (2006) Testing the accelerator hypothesis: body size, beta-cell function, and age at onset of type 1 (autoimmune) diabetes: response to Dabelea et al. Diabetes Care 29:1462-1463 (author reply 1463-1464)

13. Knowler WC, Barrett-Connor E, Fowler SE et al.; Diabetes Prevention Program Research Group (2002) Reduction in the incidence of type 2 diabetes with lifestyle intervention or metformin. N Engl J Med 346:393-403 